\title{
The role of frustration in the extinction of a running response'
}

\author{
David G. Elmes \\ UNIVERSITY OF VRGINLA
}

\begin{abstract}
Abstraet
At the end of a runway rats were partially reinforced in one goal box, to which they ran on half the trials, and were never reinforced in a different goal box, to which they ran on the remaining trials. During extinction one group ran to both goal boxes, another group ran to the reinforced goal box, and a third group ran to the nonreinforced goal box. Consistent with frustration theory, the latter group was the most resistant to extinction.
\end{abstract}

\section{Problem}

The discrimination of the transition from acquisition to extinction has been shown to be a more important determiner of the rate of extinction than the presence of secondary reinforcing stimuli during extinction (Elam et al., 1954). Elam et al. continuously reinforced rats in one goal box, to which they ran on half the trials, and never reinforced the animals in a different goal box, to which they ran on the remaining trials. In view of Amsel's $(1958,1962)$ formulations concerning frustration and the partial reinforcement effect, the present experiment was designed such that the animals were partially reinforced in the rewarded goal box. As well as consisting of the two extinction groups that Elam et al. ran (i. e., one to the reinforced goal box and one to the nonreinforced goal box), the experiment to be described included a third extinction group that ran to both goal boxes. The latter group, according to the discrimination hypothesis (Mowrer \& Jones, 1945), should be the most resistant to extinction, since acquisition and extinction would be most similar for this group. However, Amsel's frustration theory would predict that the group that ran to the nonreinforced goal box should extinguish at the slowest rate, because this group would not encounter any frustrating goal events during extinction.

\section{Method}

Twenty-eight naive, male Wistar rats served as Ss. The animals were approximately 90 days of age at the start of the experiment.

A Hunter model 380 Animal Runway and model 120A Klockounter, which recorded running time to the nearest 0.1 sec., were employed. Running time was defined as the time taken to traverse the 24-inch distance between the two photocells, one of which was located 4 inches from the start-box door, while the other was located 4 inches from the goal-box door. Retrace doors were positioned at the beginning and the end of the runway. A black flannel curtain was suspended from the end of the runway to prevent $\mathrm{S}$ from observing the interior of the goal box from the runway. Four $12 \times 7 \times 71 / 2$-inch wooden boxes, two of which had black interiors and two, white interiors, served as goal boxes. To eliminate traces of food on nonreinforced trials, one box of each color was employed on reinforced trials, and the other was used on nonreinforced trials. Secured in each goal box was a soft-drink cap which served as a food dish. The contents of the cap (45-mg Noyes pellets on reinforced trials) were concealed from the runway by a metal shield that was positioned in front of the dish.

Following adjustment to 22-hr. cyclic food deprivation, two groups matched on body weight received a total of 96 acquisition trials at an average of three trials per day. Within each block of four trials, two were run to the positive goal box, one of which was reinforced; and the remaining two trials were run to the negative goal box, neither of which was reinforced. For half of the Ss the positive goal box was black and the negative goal box was white, while white was the positive goal box and black was the negative goal box for the remaining half of the Ss.

Extinction was begun the day following the end of acquisition. Eighty-four trials were run at an average of three trials per day. The three extinction groups were matched for acquisition running time, and half the Ss in each group had been reinforced in a white goal box, while the remaining half of each group had been reinforced in a black goal box. One group of $10 \mathrm{Ss}$ ran only to the previously reinforced goal box (Group PR), another group of 10 ran only to the previously nonreinforced goal box (Group PNR), and the remaining eight Ss ran to both the previously reinforced and previously nonreinforced goal boxes (Group PR-PNR).

On all acquisition and extinction trials, $S$ was confined in the goal box for $20 \mathrm{sec}$. and then returned to his home cage for the duration of the intertrial interval, which was never less then $15 \mathrm{~min}$. Each animal was run under an approximate deprivation of $20 \mathrm{hr}$.

\section{Results}

As may be seen in Fig. 1, Group PR was running the slowest at the end of extinction, Group PNR the fastest, while Group PR-PNR was running at a speed that was between the speeds of the other two groups. These differences cannot be attributed to acquisition since analysis of variance of running time during the last 16 trials of acquisition indicated that the groups failed to differ $(F<1.00)$. That the differences among the groups were due to extinction treatments is suggested by the significant differences that were found when Groups PNR and 


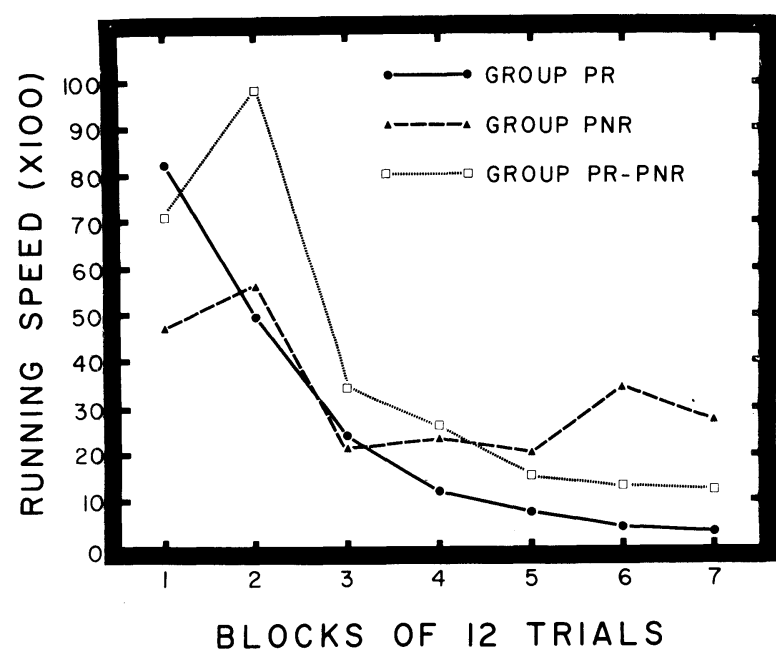

Fig. 1. Extinction running speed for each condition as a function of blocks of 12 trials.

PR-PNR and Groups PR and PR-PNR were compared separately with analyses of variance. Trend analyses of variance were also computed on these same data, and in both instances the Groups X Trials interaction was a highly significant source of variance $(p<.001)$. Thus, it may be concluded that Group PNR extinguished at the slowest rate, Group PR at the fastest rate, and that Group PR-PNR extinguished at a rate that was between the rates of the other two groups.

\section{Discussion}

That Group PNR was the most resistant to extinction is contrary to predictions based on the discrimination hypothesis but can be accounted for by frustration theory. The total number of extinction trials on which each group ran to a previously reinforced goal box seems to be the important factor determining the rate of extinction. In terms of Amsel's theory, the more often S encounters frustrating goal events (i.e., goal situations in which reinforcement that had been present on previous trials is missing) during extinction, the greater the number of occasions on which frustration-evoked competing responses will be elicited. Furthermore, repeated running to a reinforced goal box in the absence of primary reinforcement would lead to the rapid extinction of $\mathrm{r}_{\mathrm{g}}$ $s_{g}$ and $r_{f}-s_{f}$, which in turn would lead to a decrement in the running response. Group PNR was, therefore, the most resistant to extinction, because it did not encounter any frustrating goal events during extinction. Since Group PR, which extinguished most rapidly, encountered frustrating goal events on every extinction trial, it not only had the greatest number of competing responses to contend with but also suffered the largest decrement in its running response due to the rapid extinction of $r_{g}$ and $r_{f}$. Because Group PR-PNR encountered more frustrating goal events during extinction than did Group PNR but fewer than did Group PR, it extinguished at a rate that was between the rates of the other two groups.

\section{References}

AMSEL, A. The role of frustrative nonreward in non continuous reward situations. Psychol. Bull., 1958, 55, 102-119.

AMSEL, A. Frustrative nonreward in partial reinforcement and discrimination learning: some recent history and a theoretical extension. Psychol. Rev., 1962, 69, 306-328.

ELAM, C. B., TYLER, D. W., \& BITTERMAN, M. E. A further study of secondary reinforcement and the discrimination hypothesis. J.comp. physiol. Psychol., 1954, 47, 381-384.

MOWRER, O. H., \& JONES, H. Habit strength as a function of the pattern of reinforcement. J.exp. Psychol., 1945, 35, 293-311.

\section{Note}

1. This report is based upon a thesis submitted to the University of Virginia in partial fulfillment of the requirements for the degree of Honors in Psychology. The author is deeply indebted to John H. Wright for his guidance during the course of the study and to both Dr. Wright and L. Starling Reid for critically reading this manscript. Portions of these data were reported at the meeting of the Virginia Academy of Science, Charlottesville, May 1964. 